Introduction Macrophage activation syndrome (MAS) is a severe condition, which can appear as a complication of inflammatory rheumatic diseases such as systemic juvenile idiopathic arthritis (sJIA) and adult onset Still's disease (AOSD), a viral infection, or a malignancy. Interleukin (IL) -18 is a proinflammatory cytokine of the IL-1 family, known as a strong interferon (IFN)- $\gamma$ inducer, that is naturally inhibited by IL-18 binding protein (IL-18BP). High levels of unbound biologically active IL-18 have been described in patients with sJIA, AOSD, and MAS, suggesting that IL-18 is involved in the pathogenesis of these diseases.

Objectives To examine the effect of excessive IL-18 signalling in a mouse model of MAS induced by repetitive toll like receptor (TLR)9 stimulation, and explore the consequences of IL-18 or IFN- $\gamma$ blockade on MAS manifestations.

Methods MAS was induced by repeated intraperitoneal CpG injections in IL-18BP deficient $\left(I L-18 B P^{-/}\right)$mice and in wild type (WT) littermates. Anti-IL-18 receptor (IL-18R) or antiIFN- $\gamma$ monoclonal antibodies were administered prior to $\mathrm{CpG}$ injections. Clinical and biological manifestations of MAS were studied. Plasma levels of free IL-18 were measured by ELISA. Expression levels of IFN- $\gamma$ and downstream IFN- $\gamma$-induced effectors (CXCL9, CIITA) were explored by ELISA or Luminex in plasma, and by RT-qPCR in spleen and liver.

Results Naïve $I L-18 B P^{-/}$mice had no spontaneous phenotype. After repeated $\mathrm{CpG}$ injections, $I L-18 B P^{/-}$mice displayed significantly more severe MAS phenotype than their WT littermates, including more pronounced weight loss, splenomegaly, anaemia, thrombocytopenia, hyperferritinemia and hepatitis. This phenotype was associated with elevated plasma levels of unbound IL-18 and the presence of bone marrow hemophagocytes in $I L-18 B P^{-/}$mice only. In addition, $I L-18 B P^{-/}$mice displayed higher plasma levels of IFN- $\gamma$ and CXCL9, as well as increased If $n \gamma, \mathrm{Cxcl} 9$ and CIIta mRNA expression in the spleen and liver. IL-18 blockade using an anti-IL-18R antibody attenuated MAS manifestations in $I L-18 B P^{/-}$mice and abrogated IFN- $\gamma$ production and downstream signalling. IFN- $\gamma$ blockade using an anti-IFN- $\gamma$ antibody also attenuated the MAS phenotype.

Conclusions By using $I L-18 B P^{-/}$mice, we showed that unopposed IL-18 signalling was detrimental in the TLR9-induced MAS model. Importantly, blocking IL-18, as well as IFN- $\gamma$, improved disease in $I L-18 B P^{-/}$mice. Altogether, our results suggest that IL-18 exerts a pathogenic role in this model of MAS, acting upstream of IFN- $\gamma$.

Disclosure of interest None declared

\section{WHICH PLACE FOR THE NLRP3 INFLAMMASOME IN THE MSU CRYSTAL-INDUCED INFLAMMATORY RESPONSE IN VIVO?}

${ }^{1} \mathrm{~A}$ Mariotte*, ${ }^{2} \mathrm{Z}$ Zhang, ${ }^{1} \mathrm{~A}$ de Cauwer, ${ }^{3} \mathrm{~B}$ Frisch, ${ }^{1} \mathrm{~S}$ Bahram, ${ }^{2} \mathrm{R}$ Ricci, ${ }^{1} \mathrm{P}$ Georgel. 'Laboratoire d'Immuno-Rhumatologie Moléculaire - INSERM S1109, Institut d'Hématologie et d'Immunologie; ${ }^{2}$ UMR 7104 CNRS/U 964 INSERM, IGBMC, Strasbourg; ${ }^{3}$ Laboratoire de Bio-vectorologie, Faculté de pharmacie, Illkirch, France

\subsection{6/annrheumdis-2018-EWRR2018.26}

Introduction The NLRP3 (Nucleotide-binding and oligomerisation domain, Leucine-Rich repeat and Pyrin containing 3) inflammasome is a multiprotein complex made of the NLRP3 sensor, the caspase 1 enzyme and an adaptor, ASC. Upon exposure to sterile or infectious stimuli, the NLRP3 inflammasome assembles and cleaves pro-IL-1 $\beta$ and pro-IL-18 into their bioactive forms. Gout is a common microcrystalline arthritis induced by MSU (Mono-Sodium Urate) crystals accumulation which triggers an inflammatory response depending on the NLRP3-IL-1-IL-1R axis in vitro ${ }^{1}$; however, this relationship is far less understood in vivo. PKD (Protein Kinase D) were recently shown as important regulators of the NLRP3 inflammasome. ${ }^{2}$

Objectives To study the in vivo relevance of the PKDs-NLRP3 axis in two mouse models of gout.

Methods Experiments were conducted in mice deficient for NLRP3, for PKD1 and 3 or mice in which PKD1, 2 and 3 were blocked by a pan-PKD inhibitor. Peritoneal macrophages were stimulated by MSU crystals to assess IL-1 $\beta$ production. Gouty arthritis was triggered by subcutaneous injections of MSU crystals in the hind-paws and MSU-induced peritonitis by crystals injection in the peritoneal cavity.

Results We confirmed the absence of IL1 $\beta$ release by MSUchallenged peritoneal macrophages isolated from NLRP3KO animals. Pharmacological pan-PKD inhibition gave similar results, whereas genetic loss of PKD1 and 3 did not. The peritoneal gout model showed a reduction in neutrophils recruitment in NLRP3KO animals, and an increased neutrophils influx in PKD1-3dKO mice. Finally, we did not observe any alteration in gout severity in NLRP3-deficient and panPKD inhibitor-treated mice following MSU crystal subcutaneous injections; PKD1-3dKO animals even displayed more severe symptoms in this assay.

Conclusions Our data seem to confirm that the PKD-NLRP3 axis exerts a major role in sensing MSU crystals in vitro but the relevance of this pathway in our in vivo gouty arthritis models is not so clear. We speculate that an alternative pathway could compensate for the loss of IL-1 $\beta$ in NLRP3 inflammasome-deficient mice. Unravelling this possibility might be of major fundamental interest and could even lead to new therapeutics in the field of inflammation.

\section{REFERENCES}

1. Martinon F, et al. Nature 2006.

2. Zhang Z, et al. J. Exp. Med 2017.

Disclosure of interest None declared

\section{MECHANICAL STRAIN DETERMINES THE SITE-SPECIFIC DIRECTION OF INFLAMMATION AND TISSUE DAMAGE IN ARTHRITIS}

${ }^{1}$ I Cambré*, 'D Gaublomme, ${ }^{1} \mathrm{~A}$ Burssens, ${ }^{1} \mathrm{P}$ Jacques, ${ }^{1} \mathrm{D}$ Elewaut, ${ }^{2} \mathrm{R}$ Lories, ${ }^{3} \mathrm{G}$ Schett, ${ }^{4} \mathrm{G}$ Kollias, ${ }^{5} \mathrm{~A}$ de Muynck, ${ }^{6} \mathrm{~L}$ Meuris, ${ }^{6} \mathrm{~N}$ Callewaert, ${ }^{7} \mathrm{~S}$ Lambrecht, ${ }^{2} \mathrm{~S}$ Carter. ${ }^{1}$ Rheumatology, UgentNIB, Ghent; ${ }^{2}$ Skeletal biology and engineering research centre, KULeuven, Leuven, Belgium; ${ }^{3}$ Rheumatology and immunology, Friedrich Alexander University of Erlangen-Nuremberg and Universitatsklinikum, Erlangen, Germany; ${ }^{4}$ Devision of Immunology, Alexander Fleming, Attica, Greece; ${ }^{5}$ UGCT-Department of Physics and Astronomy, UGent; ${ }^{6}$ Unit of medical biotechnology, UGentVIB; ${ }^{7}$ Rheumatology, UGent, Ghent, Belgium

\subsection{6/annrheumdis-2018-EWRR2018.27}

Introduction Many pro-inflammatory pathways leading to arthritis act systemically on the immune system rather than locally in the joint. However, the reason behind the regional and patchy distribution of arthritis represents a longstanding paradox.

Objectives Explore the relation between mechanical strain and joint inflammation and understand the underlying basis of joint pattern involvement in inflammatory rheumatic diseases. 